\author{
Military Technical College \\ Kobry El-Kobbah, \\ Cairo, Egypt.
}

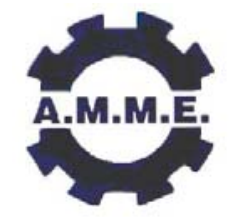

\title{
MORPHOLOGICAL CONTROL OF SINGLE CRYSTALLINE SILICON NANOWIRES AT NEAR-ROOM TEMERATURES
}

\author{
CHEN ${ }^{1} \mathrm{C}-\mathrm{Y}, \mathrm{WU}{ }^{1} \mathrm{C}-\mathrm{S}, \mathrm{CHOU}{ }^{2} \mathrm{C}-\mathrm{J}$ and YEN ${ }^{1,{ }^{*}} \mathrm{~T}-\mathrm{J}$
}

\begin{abstract}
By using a statistic electroless metal deposition (SEMD) method, here we report the morphological controllability of single crystalline silicon nanowires (SiNWs) with respect to their orientations, diameters, and lengths. The growth axis of single crystalline SiNWs on three oriented Si wafers- (100), (110) and (111)- is the [100] direction. Furthermore, the consistent results examined based on the arrays of SiNWs evidently indicate that the [100] direction is the preferential axial orientation of fabricated SiNWs in all cases. Notice that our observation is different from the previous reports, and such a corresponding formation mechanism of anisotropic SiNWs can be successfully elucidated by both lattice configuration of oriented Si surfaces and the passivation effect on the H-terminated planes. Next, the diameter control of SiNWs is achieved by employing the Taguchi methods, proving the capability of controlling the diameter with narrow distribution and comprehension of the influences from all process factors. The length of SiNWs presents fast and linear dependence with the immersion time. In addition to the morphological control of SiNWs, our statistic EMD technique provides further advantages such as almost room-temperature operation and catalyst/dopant free, paving a way towards the implementation of SiNWs in nanoelectronics, nanoscale optoelectronics, nano-electro-mechanical systems, and biological detection.
\end{abstract}

\section{KEY WORDS}

SEMD, SiNWs

1 Department of Materials Science and Engineering, National Tsing Hua University Hsinchu, 300, Taiwan (R. O. C.).

2 Department of Industrial Engineering and Engineering Management, National Tsing Hua University, Hsinchu, 300, Taiwan (R. O. C.).

* E-mail: tjyen@mx.nthu.edu.tw 


\section{INTRODUCTION}

Silicon (Si) has been dominating solid-state electronics over decades and now further leads to other applications ranging from nano/micro-electro-mechanical systems ${ }^{1}$, optoelectronics $^{2-4}$, Si Raman laser ${ }^{5}$, solar cells ${ }^{6}$ to biological and chemical sensors ${ }^{7}$. Indeed, Si-based structures are widely employed in diverse fields and among them, the common trend is to keep scaling down the device size for compact systems, enhanced performance and even revolutionary properties and therefore, currently the research regarding silicon nanowires (SiNWs) becomes a compelling topic. In fact, a crucial key to implement practical applications of SiNWs is to control their morphology in nanoscale. By using a statistic electroless metal deposition (SEMD) method, here we report the morphological controllability of single crystalline SiNWs with respect to their orientations, diameters, and lengths. We also indicate the preferential orientation is the [100] direction, different from the previous reports ${ }^{8-10}$. Compared with other growth methods, our results posses further advantages of near-room temperature process, catalyst free, and no saturated dopant contamination. So far various methods to fabricate SiNWs have been reported such as vapor-liquid-solid (VLS) growth ${ }^{11}$, vaporsolid-solid (VSS) growth ${ }^{12}$, catalytic chemical vapor deposition (CVD) ${ }^{14}$, and others ${ }^{15-17}$. Nevertheless, most methods often result in randomly oriented SiNWs, and their diameters and lengths distribute widely [ref]. More critically, SiNWs fabricated by the catalytic processes inevitably contain saturated dopants from the catalyst [ref] (especially the most popular catalyst is gold, an extremely lethal impurity for ICs) and typically the process temperature is above $800^{\circ} \mathrm{C}^{11,13,14,16}$, radically impeding their feasibility in integrated circuit industry. As a result, in this letter we utilize a statistic electroless metal deposition method to synthesize SiNWs from three oriented Si (100), (110) and (111).

\section{EXPERIMENTAL WORK}

The experiments were performed in three kinds of single crystal silicon substrates: Bdoped Si (100) (1-100 $\Omega-\mathrm{cm})$, B-doped Si (110) (1-10 $\Omega-\mathrm{cm})$ and B-doped Si (111) (1$30 \Omega-\mathrm{cm}$ ). We dipped the samples into $\mathrm{AgNO}_{3} / \mathrm{HF}$ aqueous solution (solute 0.7 grams of $\mathrm{AgNO}_{3}$ in $10 \mathrm{ml} \mathrm{Dl}$ water by adding $2 \mathrm{ml} \mathrm{HF}$ (49 wt\%) as etching agents) for $40 \mathrm{~min}$ and the corresponding galvanic reaction is as below:

$$
\begin{aligned}
& \text { Cathode }: \mathrm{Ag}^{+}+\mathrm{e}^{-} \rightarrow \mathrm{Ag} \\
& \text { Anode }: \mathrm{Si}+6 \mathrm{~F}^{-} \rightarrow \mathrm{SiF}_{6}^{2-}+4 e^{-} \\
& \text {Overall }: 4 \mathrm{Ag}^{+}+\mathrm{Si} \rightarrow 4 \mathrm{Ag}+\mathrm{SiF}_{6}^{2-}
\end{aligned}
$$

After that, we washed away the silver precipitation by nitride acid and then dried the samples.

For the estimation of the SiNWs diameters, the SiNWs were carefully scratched from samples and dispersed in ethanol under ultrasonic bath about $1 \mathrm{~min}$. Several drops of SiNWs suspension were placed on a clean holder and followed by depositing an ultrathin gold film (smaller than $2 \mathrm{~nm}$-thick) on the top in order to enhance the resolution of SEI image after drying. We carefully averaged the diameters of as-prepared SiNWs via SEM measurement from at least 150 samples for each experiment. 


\section{RESULTS AND DISCUSSIONS}

As shown in Fig. 1, the axial directions of as-synthesized SiNWs are characterized by selective area electron diffraction patterns (SAED), showing single crystalline SiNW arrays in all cases. The consistent results examined from several nanowires evidently indicate that the [100] direction is the preferential axial orientation of fabricated SiNWs among three different oriented substrates. Notice that our finding contradicts the previous works, in which no preferential crystallographic direction of SiNWs was demonstrated but always perpendicular to the surface of Si substrates regardless of the $\mathrm{Si}$ substrates used ${ }^{8-10}$. To clarify the issue of the preferential direction, we further investigate the morphologies of SiNW arrays for all three cases. First, the top view and cross-section of the as-prepared SiNWs from both Si (100) and (110) substrates are shown in Fig. 2. Referring to Fig. 2a, straight SiNWs generated on (100) substrates are always perpendicular to the etched surface, similar to the previous report ${ }^{8}$. Nevertheless, in the case of (110) substrates we routinely observe inclined SiNW arrays as shown in Fig. 2b (cross section) and Fig. 2c (top view). The red arrows, denoted the projection of the nanowires on (110) planes, are aligned along two principal axes perpendicular to one another, corresponding to the lattice configuration of [100] directions. This concluded result from large amounts of SiNWs consists with our TEM analysis examined from several SiNWs, providing sound verification of the preferential [100] orientation. Interestingly, we also find the branching pores (Fig. 2d), which can be explained by the equally preferential etch along $\langle 100\rangle$ directions.

Next, the SiNW arrays synthesized on (111) substrates appear more complicated morphology than (100) and (110) substrates. There exist two distinct directional groups, [111] and [100] SiNWs shown in Fig. 3a and Fig. 3b, respectively. Similarly we study the crystallographic projection of SiNWs and reveal that the projection on (111) planes forms a normal triangle (Fig. 3c). Such orthographic projection characterizes that these directions belong to [100] directions and agree well with the result of TEM analysis. Notice that two groups of [100] and [111] SiNWs do not distribute equally- the former dominates the latter (Fig. 3d), which can be further confirmed by measuring their average lengths $(45 \mathrm{~mm}$ for [100] SiNWs from Fig.3b and $32 \mathrm{~mm}$ for [111] SiNWs from Fig 3a). As a consequence, the preferential crystallographic orientation of SiNWs on $\mathrm{Si}$ (111) substrates remains the [100] direction as same as the cases of the other two oriented Si substrates.

The anisotropic crystallographic orientation is also applicable to the porous Si formation under HF electrolyte, which exhibits the similar pore propagation preferentially along [100] directions as well ${ }^{18-20}$. The orientation dependence can be interpreted by the lattice configuration of Si surfaces; for instance, the (100) plane presents two covalent bonds symmetrically directed into the reactive solution, leading the most sterically favored geometry to remove surface atoms along [100] directions ${ }^{18,19}$. In addition to the lattice configuration, the preferential direction [100] can be also explained by the effect of the passivation on hydrogen $(\mathrm{H})$-terminated silicon atoms ${ }^{21}, 22$. The velocity of $\mathrm{H}$ termination on (111) crystallographic planes is greater than other planes (i.e., (111) planes become etching-stop planes), and thus their correlated direction [100] allows the etching reaction to propagate along this preferential orientation. On the other hand, the anisotropic propagation without lateral etching was explained previously by the shortest charge-transport distance, occurring at the pore tips rather than on the pore sidewalls ${ }^{9}$. Yet, this suggestion seemed not convincible because the charge-transport distance between $\mathrm{Ag}$ particles and $\mathrm{Si}$ atoms is similar at both locations. Instead, based on our 
observation and explanation aforementioned, one can obtain a straightforward comprehension for this anisotropic phenomenon.

In addition to the oriented growth of SiNWs, another key of realizing practical devices based on SiNWs is to control their diameters and lengths ${ }^{23}$. Basically the conventional EMD method lacks control on diameters because of multiple process factors involved in its galvanic reaction simultaneously so that the diameter of formed SiNWs distributes widely from 30 to $300 \mathrm{~nm}$ [Ref]. Recently, a nanosphere lithographic process appeared to improve the controllability on the diameters of $\mathrm{SiNWs}^{24}$, but the overall process eventually became more complicated against the advantage of using the EMD method. Therefore, we return to examine the original EMD process and apply Taguchi methods, a well-established design of experiments for engineering productivity and quality control $^{25}$. We design an L9 orthogonal array of experiments as shown in Table 1, dealing with four critical process factors including reaction temperature $(A)$, concentrations of $\mathrm{AgNO}_{3}(\mathrm{~B})$ and $\mathrm{HF}(\mathrm{C})$, and immersion time (D). Each factor varies in three different levels and the total degrees of freedom are eight. In this study, the (100) silicon substrate is chosen for the purpose of eliminating the consideration of orientation dependence.

Based on the experimental results of Taguchi methods, a subsequent analysis of variance (ANOVA) is conducted to identify the individual significance of those four process factors. For example, a designed diameter of SiNWs is set to be $100 \mathrm{~nm}$, so the optimal levels of each process factor are determined as $A_{2} B_{2} C_{2} D_{2}$ analyzed through the $\mathrm{S} / \mathrm{N}$ ratio (signal-to-noise). To assure the accuracy of the suggested optimal levels, we further conduct a confirmatory experiment. Fig. 4a plots the distributed diameters of SiNWs fitted by Gaussian curves from the confirmatory experiment (i.e., the target is $100 \mathrm{~nm}$ ) and all the original nine trials. Remarkably, the mean diameter of SiNWs via the confirmatory experiment presents $101.75 \mathrm{~nm}$, very close to our target value. More importantly, compared with the original nine trials (Table 1), now the standard deviation in diameters is significantly reduced to $21.33 \mathrm{~nm}$ that indicates a stable and reliable result of optimized factor levels. Besides, we calculate the degrees of individual contributions from each factor ordered by their significance- the concentration of HF (41.35\%), the concentration of $\mathrm{AgNO}_{3}(26.672 \%)$, the reaction temperature $(19.576 \%)$, and the immersion time whose trivial contribution can be ignored. Actually the results of the diameter control analyzed by Taguchi methods also guide us to target different designed diameters; that is, as the diameter is designed to be $150 \mathrm{~nm}$, we should first dilute the concentrations of $\mathrm{HF}$ as well as $\mathrm{AgNO}_{3}$. In short, we report a stable and reliable method to control the diameter of SiNWs, in which the concentration of HF (C) impacts foremost but the immersion time (D) is almost independent with the diameter of SiNWs.

Finally, we focus on controlling the lengths of SiNWs. In order to maintain the uniform diameters of SiNWs, we adopt the optimized factor levels aforementioned (target $=100$ $\mathrm{nm}$ ) but vary the immersion time from 1, 5, 10 to 15 minutes, respectively. Fig. 4 displays a linear relationship between the lengths of SiNWs versus the immersion time and the corresponding cross-sectional SEM images of as-prepared SiNWs arrays. It is clear that SiNWs grow significantly fast with a constant rate (about $1 \mathrm{~mm} / \mathrm{min}$ ) and meanwhile all nanowires are fashioned uniformly through the entire sample. In a sense, such linear, fast, and uniform growth behavior facilitates the length control of SiNWs in a large scale, and can be easily utilized to achieve high-aspect-ratio SiNWs at the room temperature in a short period (e.g., the ratio achieves 150 in $15 \mathrm{~min}$ ). 


\section{CONCLUSION}

In this letter we have demonstrated large-scale single crystalline SiNW arrays with the morphological control of their orientations, diameters and lengths by a statistic EMD technique. The preferential crystallographic orientation of fabricating SiNWs is the [100] direction, proved by both TEM diffraction patterns and the orthographic projections on three oriented Si substrates. The formation mechanism of anisotropic SiNWs can be successfully elucidated in accordance with both the lattice configuration of oriented $\mathrm{Si}$ surfaces and the passivation effect on the $\mathrm{H}$-terminated planes. The diameter control of SiNWs is achieved by employing the Taguchi methods, promising the capability of controlling the diameter with narrow distribution and comprehension of the influences from all process factors. The length of SiNWs presents fast and linear dependence with the immersion time. In addition to the morphological control of SiNWs, our statistic EMD technique provides further advantages such as almost room-temperature operation and catalyst/dopant free, paving a way towards the implementation of SiNW-based devices in nanoelectronics, nanoscale optoelectronics, nano-electro-mechanical systems, and biological detection.

\section{ACKNOWLEDGEMENT}

The authors would like to thank the National Nano Device Laboratories (NDL-94SC142) and the National Science Council (NSC 95-2112-M-007 -048 MY3) for financially supporting this study.

\section{REFERENCES}

1. Madou, M. Fundamentals of Microfabrication: The Science of Miniaturization. (CRC Press, Boca Raton, Florida, 2002).

2. Pavesi, L. Routes toward silicon-based lasers. Mater. Today 8, 18-25 (2005).

3. Yu, D. P. B., Z. G. Wang, J. J. Zou, Y. H. Qian, W. Fu, J. S. Zhang, H. Z. Ding, Y.Xiong, G. C. You, L. P. Xu, J. Feng, S. Q. Direct evidence of quantum confinement from the size dependence of the photoluminescence of silicon quantum wires. Phys. Rev. B: Condens. Matter 59, R2498-R2501 (1999).

4. Becker, M. S., V. Andra, G. Geiger, R. Schreiber, J. Hoffmann, S. Michler, J. Milenin, A. P. Werner, P. Christiansen, S. H. The SERS and TERS effects obtained by gold droplets on top of Si nanowires. Nano Lett. 7, 75-80 (2007).

5. Rong, H. S. J., R. Liu, A. S. Cohen, O. Hak, D. Fang, A. Paniccia, M. A continuouswave Raman silicon laser. Nature 433, 725-728 (2005).

6. Bozhi Tian, X. Z., Thomas J. Kempa, Ying Fang, Nanfang Yu, Guihua Yu, Jinlin Huang, Charles M. Lieber Coaxial silicon nanowires as solar cells and nanoelectronic power sources. Nature 449, 885-890 (2007).

7. Cui, Y., Wei, Q. Q., Park, H. K. \& Lieber, C. M. Nanowire nanosensors for highly sensitive and selective detection of biological and chemical species. Science 293, 1289-1292 (2001).

8. Peng, K. Q., Yan, Y.J., Gao, S.P. \& Zhu, J. Synthesis of large-area silicon nanowire arrays via self-assembling nanoelectrochemistry. Adv. Mater. 14, 11641167 (2002).

9. Peng, K.Q. H., J. J. Yan, Y. J. Wu, Y. Fang, H. Xu, Y. Lee, S. T. Zhu, J. Fabrication 
of single-crystalline silicon nanowires by scratching a silicon surface with catalytic metal particles. Adv. Funct. Mater. 16, 387-394 (2006).

10. Peng, K. Q. F., H. Hu, J. J. Wu, Y. Zhu, J. Yan, Y. J. Lee, S. Metal-particleinduced, highly localized site-specific etching of $\mathrm{Si}$ and formation of singlecrystalline Si nanowires in aqueous fluoride solution. Chem. Eur. J. 12, 7942-7947 (2006).

11. Wu, Y.Y. Yang, P.D. Direct observation of vapor-liquid-solid nanowire growth. J. Amer. Chem. Soc. 123, 3165-3166 (2001).

12. Y. Wang, V.S., Stephan Senz, Ulrich Go" Sele, Epitaxial growth of silicon nanowires using an aluminium catalyst. Nature Nanotechnology 1, 186-189 (2006).

13. Zhang, R.Q., Lifshitz, Y. \& Lee, S.T. Oxide-assisted growth of semiconducting nanowires. Adv. Mater. 15, 635-640 (2003).

14. Ge, S. P. J., K. L. Lu, X. X. Chen, Y. F. Wang, R. M. Fan, S. S. Orientationcontrolled growth of single-crystal silicon-nanowire arrays. Adv. Mater. 17, 56-61 (2005).

15. Yu, D. P. L., C. S. Bello, I. Sun, X. S. Tang, Y. H. Zhou, G. W. Bai, Z. G. Zhang, Z. Feng, S. Q. Synthesis of nano-scale silicon wires by excimer laser ablation at high temperature. Solid State Commun. 105, 403-407 (1998).

16. Yan, H. F. X., Y. J. Hang, Q. L. Yu, D. P. Wang, Y. P. Xu, J. Xi, Z. H. Feng, S. Q. Growth of amorphous silicon nanowires via a solid-liquid-solid mechanism. Chem. Phys. Lett. 323, 224-228 (2000).

17. Holmes, J. D., Johnston, K. P., Doty, R. C. \& Korgel, B. A. Control of thickness and orientation of solution-grown silicon nanowires. Science 287, 1471-1473 (2000).

18. Smith, R. L. \& Collins, S.D. Porous Silicon Formation Mechanisms. J. Appl. Phys. 71, R1-R22 (1992).

19. Lehmann, V. The Physics of Macropore Formation in Low Doped N-Type Silicon. J. Electrochem. Soc. 140, 2836-2843 (1993).

20. Chuang, S. F., Collins, S. D. \& Smith, R. L. Preferential Propagation of Pores During the Formation of Porous Silicon - a Transmission Electron-Microscopy Study. Appl. Phys. Lett. 55, 675-677 (1989).

21. Christophersen, M., Carstensen, J., Feuerhake, A. \& Foll, H. Crystal orientation and electrolyte dependence for macropore nucleation and stable growth on p-type Si. Mater. Sci. Eng., B 69, 194-198 (2000).

22. Christophersen, M. et al. Crystal orientation dependence and anisotropic properties of macropore formation of p- and n-type silicon. J. Electrochem. Soc. 148, E267E275 (2001).

23. Li, Z. et al. Silicon nanowires for sequence-specific DNA sensing: device fabrication and simulation. Appl. Phys. A 80, 1257-1263 (2005).

24. Huang, Z.P., Fang, H. \& Zhu, J. Fabrication of silicon nanowire arrays with controlled diameter, length, and density. Adv. Mater. 19, 744-+ (2007).

25. Peace, G.S. Taguchi Methods: A Hands-On Approach. (Addison-Wesley Pub. Co., Reading, MA 1993). 
Table 1 Statistic analysis of affecting factors on controlling SiNWs diameters. The left tabulates four control factors and their three levels. The right denotes the L9 orthogonal array of experiments. The average diameters and standard deviation of SiNWs are measured from at least 150 samples for each trial.

\begin{tabular}{ccccc}
\hline $\begin{array}{c}\text { Control } \\
\text { Factors }\end{array}$ & Labels & $\begin{array}{c}\text { Level } \\
\mathbf{1}\end{array}$ & Level 2 & Level 3 \\
\hline $\begin{array}{c}\text { Temperature } \\
\left({ }^{\circ} \mathrm{C}\right)\end{array}$ & $\mathrm{A}$ & 30 & 50 & 70 \\
$\mathrm{AgNO}_{3}(\mathrm{M})$ & $\mathrm{B}$ & 0.01 & 0.02 & 0.04 \\
$\mathrm{HF}(\mathrm{M})$ & $\mathrm{C}$ & 4.6 & 7.9 & 10.4 \\
$\mathrm{Time}(\mathrm{min})$ & $\mathrm{D}$ & 20 & 40 & 60 \\
\hline
\end{tabular}

\begin{tabular}{ccccccc}
$\begin{array}{c}\text { Fact } \\
\text { ors }\end{array}$ & A & B & C & D & $\begin{array}{c}\text { Average } \\
\text { diameters } \\
\text { (nm) }\end{array}$ & $\begin{array}{c}\text { Standard } \\
\text { deviation } \\
\text { (nm) }\end{array}$ \\
\hline $\begin{array}{c}\text { Trial } \\
\text { no. }\end{array}$ & & & & & & \\
\hline $\mathbf{1}$ & 1 & 1 & 1 & 1 & 254.46 & 29.15 \\
$\mathbf{2}$ & 1 & 2 & 2 & 2 & 101.25 & 32.69 \\
$\mathbf{3}$ & 1 & 3 & 3 & 3 & 152.34 & 54.92 \\
$\mathbf{4}$ & 2 & 1 & 2 & 3 & 113.65 & 38.74 \\
$\mathbf{5}$ & 2 & 2 & 3 & 1 & 105.05 & 34.61 \\
$\mathbf{6}$ & 2 & 3 & 1 & 2 & 153.59 & 53.06 \\
$\mathbf{7}$ & 3 & 1 & 3 & 2 & 176.49 & 43.22 \\
$\mathbf{8}$ & 3 & 2 & 1 & 3 & 164.93 & 50.73 \\
$\mathbf{9}$ & 3 & 3 & 2 & 1 & 146.97 & 54.24 \\
\hline
\end{tabular}



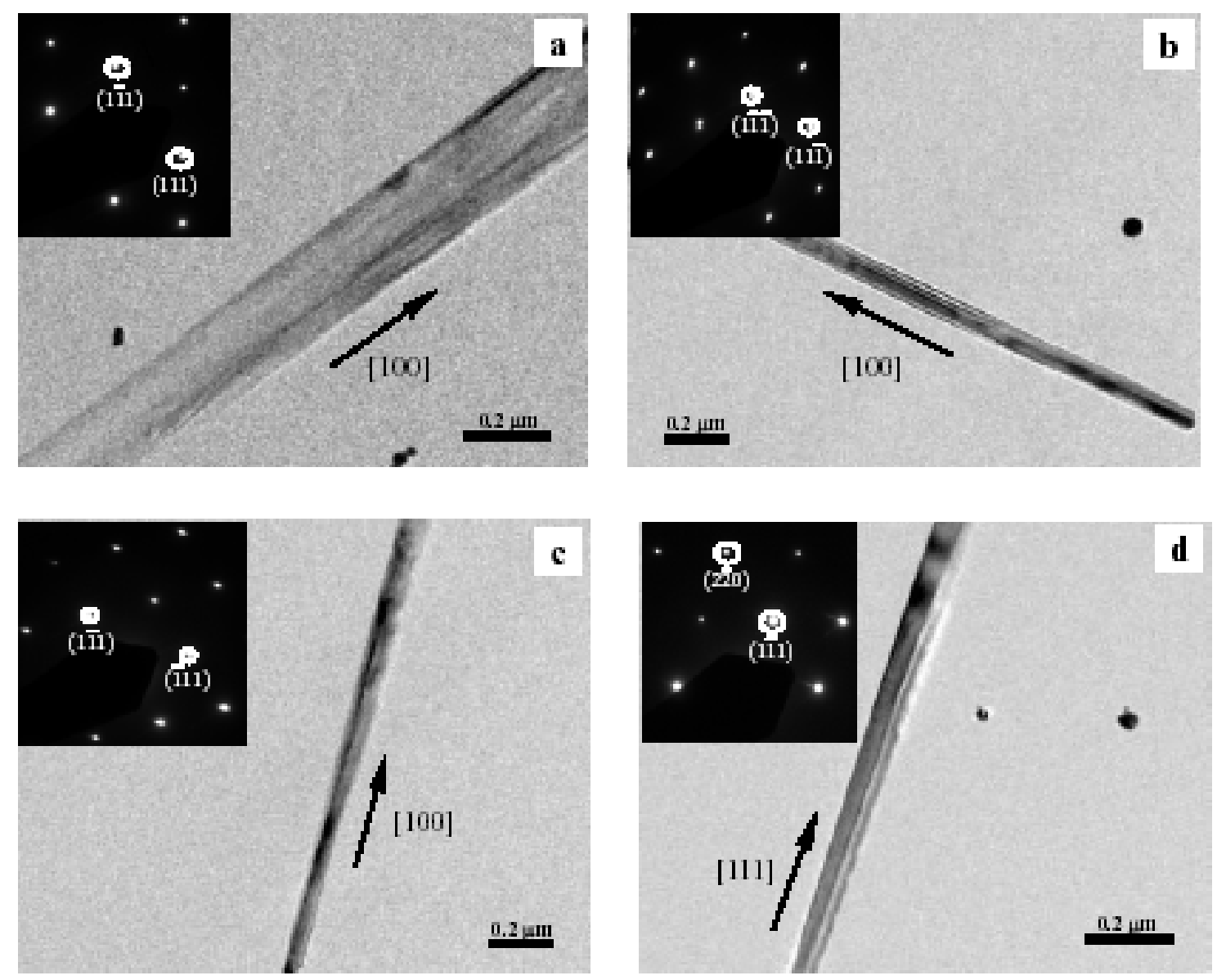

Figure 1. Characterization of the SiNWs fabricated by SEMD method. TEM images of as-prepared SiNWs fabricated on a) Si (100) substrates, b) $\mathrm{Si}$ (110) substrates, c) and d) Si (111) substrates, respectively. The products of nanowires axially aligned on [100] directions always exist on $\mathrm{Si}$ (100) and Si (110) substrates. Nevertheless, there exist two directional groups, [100] and [111] SiNWs on Si (111) substrates and the former belongs to the majority. The results evidently reveal that the preferential orientation of fabricating SiNWs is [100] direction among three different oriented substrates The insets show the corresponding SAED patterns taken from SiNWs with the zone axis along [110], indicating only single crystalline nanowires can be observed regardless of wafer orientation. 

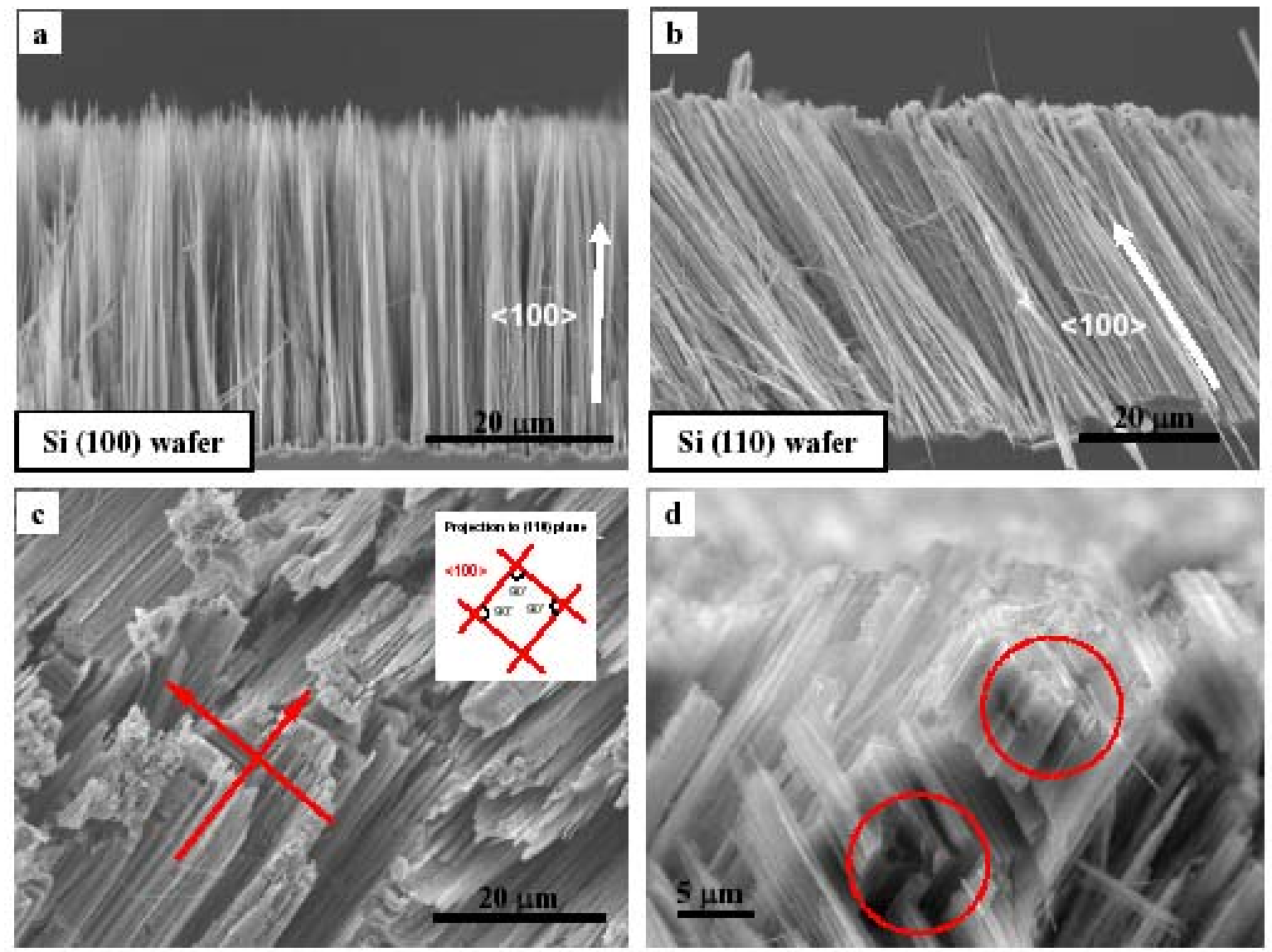

Figure 2. Investigation of preferential orientations of well-aligned SiNWs arrays on two oriented $\mathrm{Si}(100)$ and (110) substrates. Cross-sectional SEM images of SiNWs formed on a Si (100) substrates and b Si (110) substrates. c Top view of SEM image of as-grown SiNWs array on Si (110) substrates. The inset shows the projection of aligned SiNWs to a (110) plane. The orthogonal directions indicate the SiNWs oriented to [100] directions. d Cross-sectional SEM image points the branching pores on Si (110) substrates. 

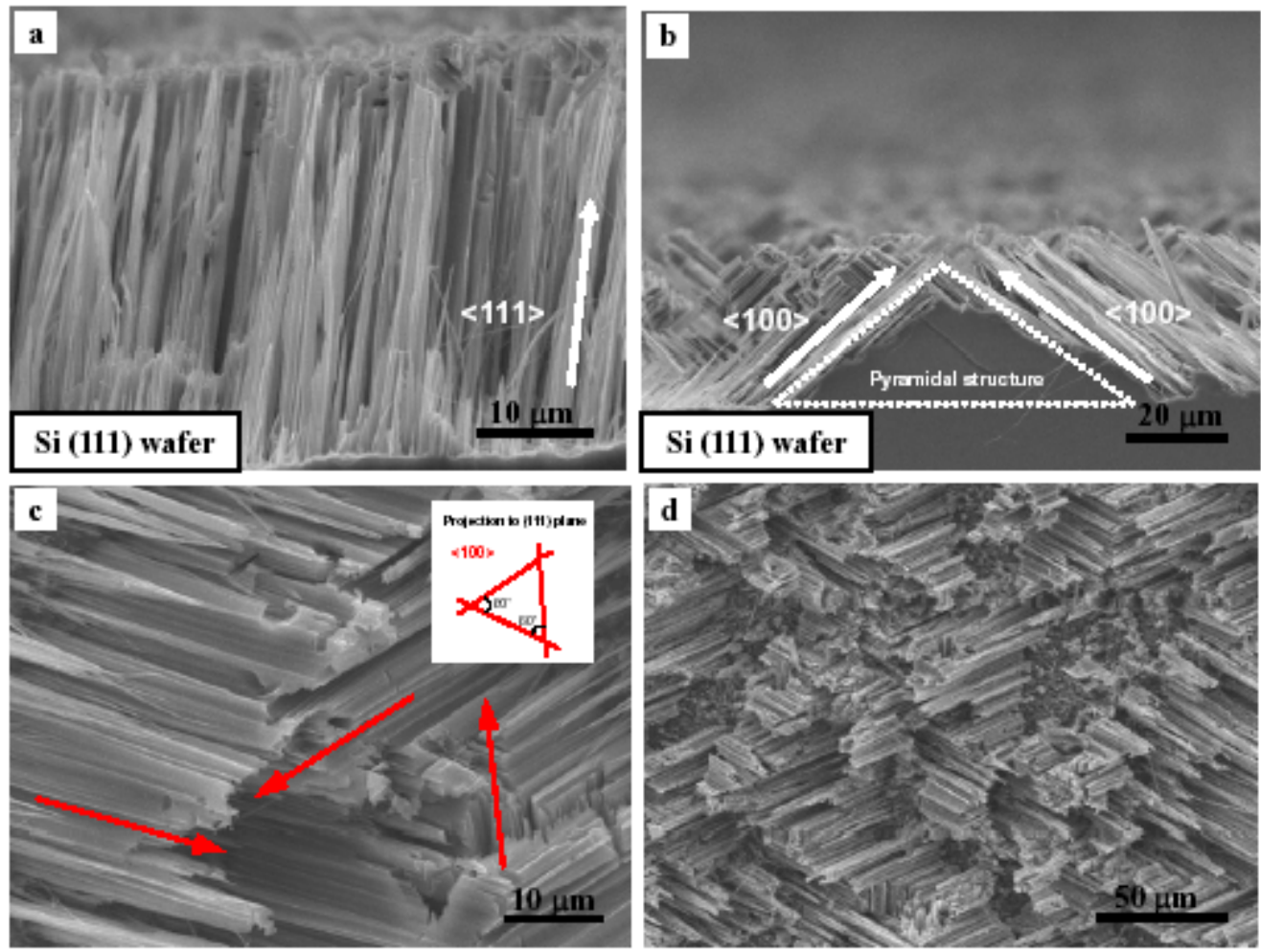

Figure 3. Investigation of preferential orientations of well-aligned SiNWs arrays on oriented Si (111) substrates. $\mathbf{a}$ and $\mathbf{b}$, Cross-sectional SEM images of SiNWs formed on Si (111) substrates. Interestingly, the pyramidal structures accompanied with the formation of [100] directional nanowires can be observed (Fig. b). These particular regions are formed under [100] anisotropic etching (The details are described in the Supplementary Information). c, Top view of SEM image of as-grown SiNWs array on Si (111) substrates. The inset shows the projection of aligned SiNWs to a (111) plane. The alignments are arranged to the normal triangle, indicating the SiNWs oriented to [100] directions. d, SEM image of final product in top view. It indicates the [100] SiNWs are preferentially formed on Si (111) substrates. 
a

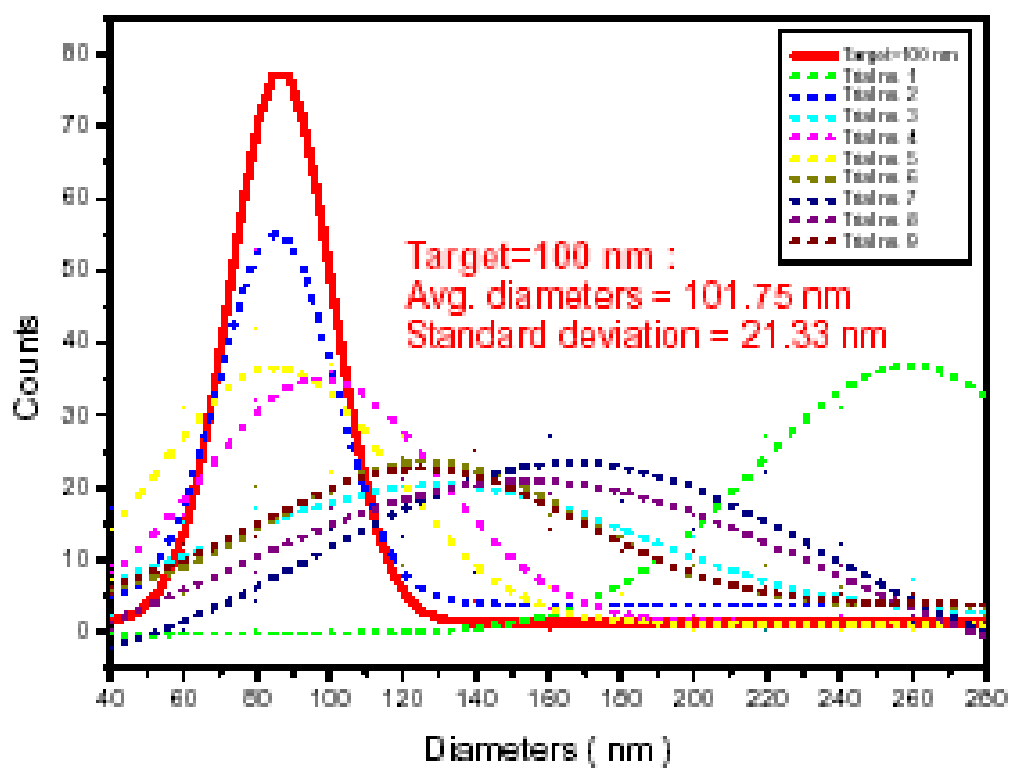

b

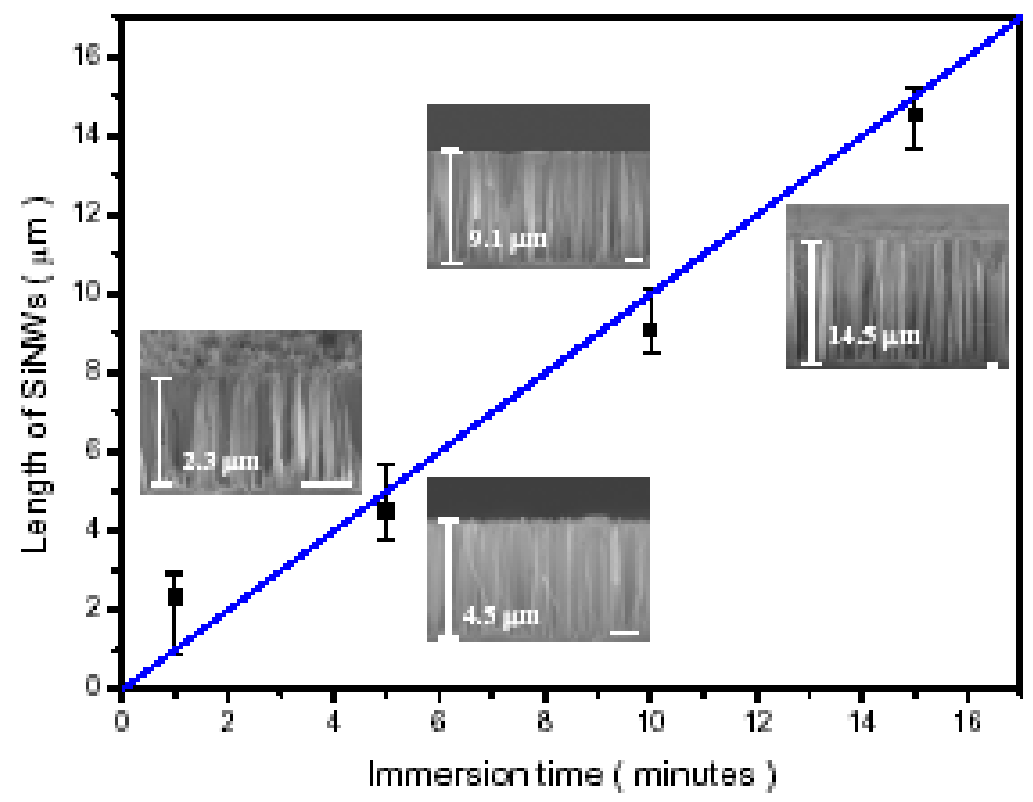

Figure 4 Controllability of SiNWs on diameters and lengths a, Gaussian fittings of the diameter distributions for confirmatory (target=100 nm) and original experiments, respectively. Indeed, the optimized process factors provide the controllability of diameters and significantly reduce the standard deviation. $\mathbf{b}$, The linear relationship between the immersion time and the length of SiNW arrays (The scale bar is $1 \mathrm{~mm}$ ). Note that the growth rate of SiNWs is about $1 \mathrm{~mm} / \mathrm{min}$. 